\title{
A Component Prediction Method for Flue Gas of Natural Gas Combustion Based on Nonlinear Partial Least Squares Method
}

\author{
Hui Cao, ${ }^{1}$ Xingyu Yan, ${ }^{1}$ Yaojiang Li, ${ }^{1}$ Yanxia Wang, ${ }^{1}$ Yan Zhou, ${ }^{2}$ and Sanchun Yang \\ ${ }^{1}$ State Key Laboratory of Electrical Insulation and Power Equipment, School of Electrical Engineering, \\ Xian Jiaotong University, Xian 710049, China \\ ${ }^{2}$ School of Energy \& Power Engineering, Xian Jiaotong University, Xian 710049, China \\ Correspondence should be addressed to Yan Zhou; yan.zhou@mail.xjtu.edu.cn
}

Received 25 December 2013; Accepted 10 February 2014; Published 19 March 2014

Academic Editors: S. Lee and J. Shiea

Copyright (C) 2014 Hui Cao et al. This is an open access article distributed under the Creative Commons Attribution License, which permits unrestricted use, distribution, and reproduction in any medium, provided the original work is properly cited.

\begin{abstract}
Quantitative analysis for the flue gas of natural gas-fired generator is significant for energy conservation and emission reduction. The traditional partial least squares method may not deal with the nonlinear problems effectively. In the paper, a nonlinear partial least squares method with extended input based on radial basis function neural network (RBFNN) is used for components prediction of flue gas. For the proposed method, the original independent input matrix is the input of RBFNN and the outputs of hidden layer nodes of RBFNN are the extension term of the original independent input matrix. Then, the partial least squares regression is performed on the extended input matrix and the output matrix to establish the components prediction model of flue gas. A near-infrared spectral dataset of flue gas of natural gas combustion is used for estimating the effectiveness of the proposed method compared with PLS. The experiments results show that the root-mean-square errors of prediction values of the proposed method for methane, carbon monoxide, and carbon dioxide are, respectively, reduced by $4.74 \%, 21.76 \%$, and $5.32 \%$ compared to those of PLS. Hence, the proposed method has higher predictive capabilities and better robustness.
\end{abstract}

\section{Introduction}

Gas-fired generator usually uses the natural gas with high calorific value to generate electricity [1]. Since the natural gasfired generator is more cost-effective and has less emission compared with coal-fired generator, it is considered as a new type of generator that gives a powerful response to the demand of environmental protection and new market environment. China has abundant domestic natural gas reserves, and the prospect of natural gas generator is bright and broad. Flue gas produced by the natural gas generator mainly consists of methane $\left(\mathrm{CH}_{4}\right)$, carbon monoxide $(\mathrm{CO})$, and carbon dioxide $\left(\mathrm{CO}_{2}\right)$ that will pollute the atmosphere under a certain extent [2]. Therefore, the prediction of components of the flue gas could reflect the degree of the possible impact on the environment and is of great significance for energy saving and emission reduction.

Traditional quantitative analysis of flue gas is always performed by the regular chemical experiment method, which is slow and cumbersome in analysis and may be limited for further application in continuous monitoring of flue gas. Near-infrared spectroscopy (NIR) technology is an effective and rapid quantitative detection method for determining the chemical components based on the characteristic spectrum. NIR has been adopted in medical affairs, food, industries, and other fields [3-6], and it can also be used to realize the component prediction of flue gas. Partial least squares (PLS) is a widely used method in NIR quantitative analysis. PLS synthetically extracts the information for the independent variables and determines the latent variables which have the best interpretability for regression modeling [7]. Since PLS could deal with the multiple correlation among the variables, the accuracy of the regression model would be improved [810]. Nevertheless, PLS is essentially a kind of linear regression and may not solve the nonlinear problems effectively [11, 12]. Nonlinear PLS (NPLS) with extended input could solve the problem. For the method, the nonlinear-related independent input matrix is extended to include the nonlinear terms of 
the independent variables (such as the square terms and the cross-product terms). Then, the PLS regression is performed on the extended input matrix of independent variables [13-15]. Because the spectral data often have thousands of wavelength variables, the extension of nonlinear terms would make the input matrix tremendously large and the nonlinear terms selection also lacks the theoretical basis. Hence, the method is restricted in the application of spectral quantitative analysis. Neural network could approximate a nonlinear function in an arbitrary precision [16-18] and could be used for the extension of the input matrix.

In this paper, a component prediction method which combines the neural network and NPLS with extended input is proposed for flue gas of natural gas combustion. The proposed method uses radial basis function neural network (RBFNN) to extend the input matrix. The original independent input matrix is the input of RBFNN and the outputs of hidden layer nodes of RBFNN are the extension term of the original independent input matrix. Then, the PLS regression is performed on the extended input matrix and the output matrix to establish the NPLS model based on RBFNN extending input (RBFEI-PLS). In order to verify the effectiveness of the proposed method, PLS and RBFEI-PLS are used for building the components quantitative analysis models of the NIR spectral dataset of flue gas of natural gas combustion and the experiments results are analyzed. The organization of the paper is as follows. Section 2 presents the proposed method. The experimental process is provided and the experiments results are discussed in Section 3. Finally, Section 4 concludes the paper.

\section{Component Prediction Method}

For the NIR spectral dataset of flue gas of natural gas combustion, the absorbance of each wavelength is the independent input $\mathbf{X}$ and the concentration of the component of flue gas is the dependent output $\mathbf{Y}$.

RBFEI-PLS uses typical three-layer construction of RBFNN to extend the input matrix $\mathbf{X}$. $\mathbf{X}$ is the input of RBFNN and the outputs of hidden layer nodes of RBFNN are the extension term $\mathbf{G}$, so the extended input matrix will be $\mathbf{X}^{\prime}=\left[\begin{array}{lll}\mathbf{X} & \mathbf{G} & \mathbf{1}\end{array}\right]$.

The $i$ th node input of the hidden layer, $g_{i}$, is shown as

$$
g_{i}(x)=\exp \left(-\frac{\left\|x_{j}-c_{i}\right\|^{2}}{\sigma_{i}^{2}}\right),
$$

where $c_{i}$ is the vector of the $i$ th node center and can be obtained according to the cluster center after clustering analysis on the spectral dataset [19]. $\sigma_{i}$ is the corresponding width parameter and equals the root mean square distance of the nearest $m$ hidden layer nodes from the $i$ th node center.

PLS regression is performed on the extended input matrix $\mathbf{X}^{\prime}$ and the output matrix $\mathbf{Y}$ with the flue gas spectral training samples. Then, the quantitative analysis model of the flue gas spectral dataset can be obtained and is shown as

$$
\mathbf{Y}=\mathbf{X A}+\mathbf{G H}+\mathbf{b}^{T},
$$

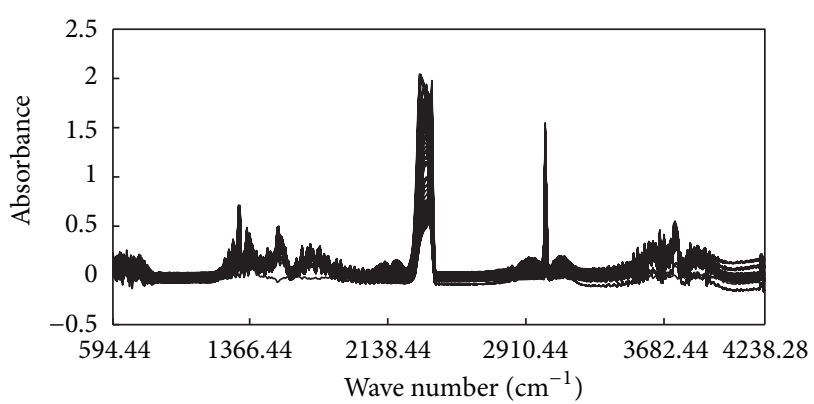

FIGURE 1: Original spectra data of fuel gas.

where $\mathbf{A}$ is the linear weighting matrix of $\mathbf{X}, \mathbf{H}$ is the nonlinear weighting matrix of the outputs of the hidden layer nodes of RBFNN, and $\mathbf{b}$ is the bias vector.

\section{Experiments Results}

3.1. Experiment Data. To evaluate the effectiveness of the proposed method, a real dataset obtained by measuring the NIR spectra of the field flue gas is used in the experiments. The dataset is obtained during a combustion process and includes 106 samples. Each sample consists of a spectrum for a mixture of $\mathrm{CH}_{4}, \mathrm{CO}$, and $\mathrm{CO}_{2}$. The concentration ranges of the three components, obtained via gas chromatograghy, are 0 $0.4598 \mathrm{ppm}, 0 \sim 0.4083 \mathrm{ppm}$, and $0 \sim 0.3818 \mathrm{ppm}$, respectively. The spectra were measured by a GASMET DX4000 Fourier transform infrared gas analyzer. The spectral wave number is $549.44 \sim 4238.28 \mathrm{~cm}^{-1}$ with a resolution of $7.72 \mathrm{~cm}^{-1}$. The original spectra are shown in Figure 1.

3.2. Experiment Method. The experiment adopts the shutters grouping strategy to divide the original spectral dataset into the calibration set and the validation sets [20] that lets the concentrations of each component vary in roughly the same range. One sample is selected into the validation set every four samples and the rest of the samples are selected into the calibrating set; namely, there are 84 samples in the calibrating set and 21 samples in the validation set. The calibration set is used for building the prediction model of PLS and RBFEIPLS, and the validation set is used for estimating the effectiveness. For RBFEI-PLS, the number of nodes of the hidden layer is 3 , and $m$ is set to 3 . For PLS and RBFEI-PLS, the number of latent variables is determined according to the root-meansquare error of five-fold cross-validation (RMSECV). In the study, RMSECV, the squared cross-validation correlation coefficient $\left(R_{\mathrm{cv}}^{2}\right)$, the root mean-squared error of calibration (RMSEC), the squared correlation coefficient of calibration $\left(R_{c}^{2}\right)$, the root-mean-square error of prediction (RSMEP), and the squared correlation coefficient of prediction $\left(R_{p}^{2}\right)$ are used to compare the predictive ability of various models.

3.3. Results and Analysis. Table 1 is the experiments results for $\mathrm{CH}_{4}$ of the flue gas dataset. Although the RMSECV of PLS is smaller, other indexes in Table 1 show that the effectiveness of RBFEI-PLS is much better than that of PLS, especially that 
TABLE 1: Experiment results for $\mathrm{CH}_{4}$.

\begin{tabular}{lcc}
\hline & PLS & RBFEI-PLS \\
\hline RMSECV & $\mathbf{2 4 3 . 7 9}$ & 324.28 \\
$R_{\mathrm{cv}}^{2}$ & 0.7693 & $\mathbf{0 . 9 7 7 8}$ \\
RMSEC & 81.1883 & $\mathbf{6 1 . 8 2 4 3}$ \\
$R_{c}^{2}$ & 0.997 & $\mathbf{0 . 9 9 8 3}$ \\
RMSEP & 99.9311 & $\mathbf{9 5 . 1 9 7 4}$ \\
$R_{p}^{2}$ & 0.9960 & $\mathbf{0 . 9 9 6 3}$ \\
\hline
\end{tabular}

TABLE 2: Experiment results for CO.

\begin{tabular}{lcc}
\hline & PLS & RBFEI-PLS \\
\hline RMSECV & $\mathbf{2 4 1 . 1 2}$ & 250.77 \\
$R_{\mathrm{cv}}^{2}$ & $\mathbf{0 . 9 3 5 8}$ & 0.9354 \\
RMSEC & 40.3678 & $\mathbf{2 8 . 2 2 6 7}$ \\
$R_{c}^{2}$ & 0.9959 & $\mathbf{0 . 9 9 8 0}$ \\
RMSEP & 75.5044 & $\mathbf{5 9 . 0 7 5 3}$ \\
$R_{p}^{2}$ & 0.98630 & $\mathbf{0 . 9 9 0 6}$ \\
\hline
\end{tabular}

TABLE 3: Experiment results for $\mathrm{CO}_{2}$.

\begin{tabular}{lcc}
\hline & PLS & RBFEI-PLS \\
\hline RMSECV & 241.12 & $\mathbf{1 5 4 . 1 2}$ \\
$R_{c v}^{2}$ & 0.5010 & $\mathbf{0 . 9 8 9 5}$ \\
RMSEC & 42.5139 & $\mathbf{2 1 . 7 7 5 9}$ \\
$R_{c}^{2}$ & 0.9982 & $\mathbf{0 . 9 9 9 5}$ \\
RMSEP & 69.5170 & $\mathbf{6 5 . 8 1 7 1}$ \\
$R_{p}^{2}$ & 0.9965 & $\mathbf{0 . 9 9 6 8}$ \\
\hline
\end{tabular}

the RMSEP value of RBFEI-PLS is $4.74 \%$ lower than that of PLS. Figure 2 shows the scatter plots of measured value versus predicted value of PLS and RBFEI-PLS for $\mathrm{CH}_{4}$. PLS has larger error and most points are distributed on both sides of the diagonal line shown in Figure 2(a). Almost all of the points of RBFEI-PLS are in the diagonal line as Figure 2(b) shows. So the prediction capability of RBFEI-PLS is higher.

Table 2 is the experiments results for $\mathrm{CO}$ of the flue gas dataset. Although the RMSECV value of PLS is smaller and $R_{\text {cv }}^{2}$ of PLS is larger, RBFEI-PLS outperforms PLS according to RMSEC, $R_{c}^{2}$, RSMEP, and $R_{p}^{2}$, where the RMSEP value of RBFEI-PLS is $21.76 \%$ lower than that of PLS. Figure 3 shows the scatter plots of measured value versus predicted value of PLS and RBFEI-PLS for CO. Considerable errors in PLS method are shown in Figure 3(a) as most points are relatively far from the diagonal line, and some points even break away from the diagonal line. Figure 3(b) indicates that the points are mostly distributed closely on both sides of the diagonal line. Therefore, the prediction model of RBFEI-PLS is still more accurate.

Table 3 is the experiments results for $\mathrm{CO}_{2}$ of the flue gas dataset. All the indexes of RBFEI-PLS are better than those of PLS, and the RMSEP value of RBFEI-PLS is 5.32\% lower than that of PLS. Figure 4 shows the scatter plots of measured

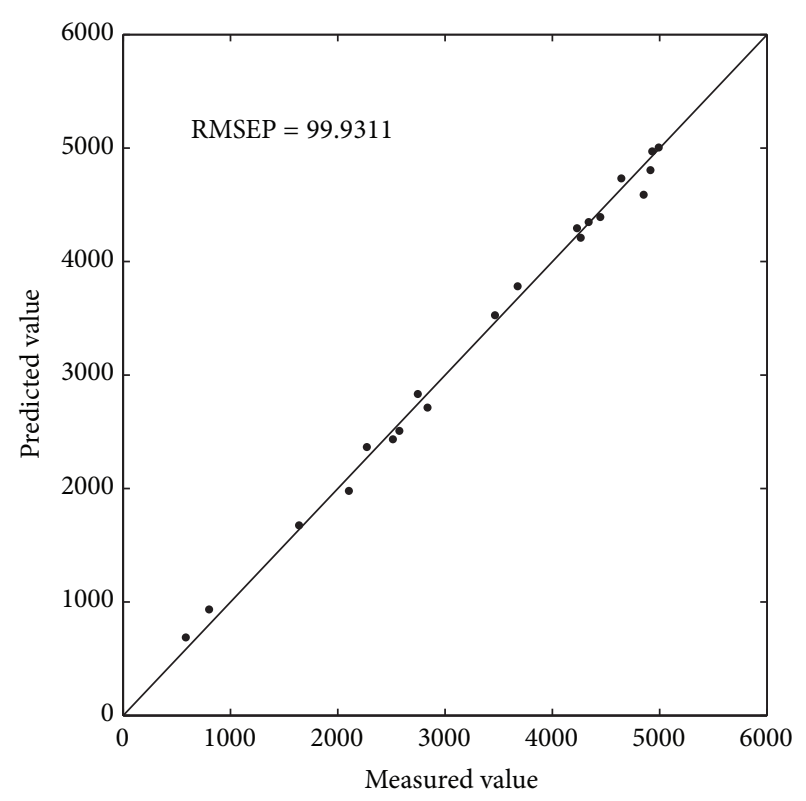

(a)

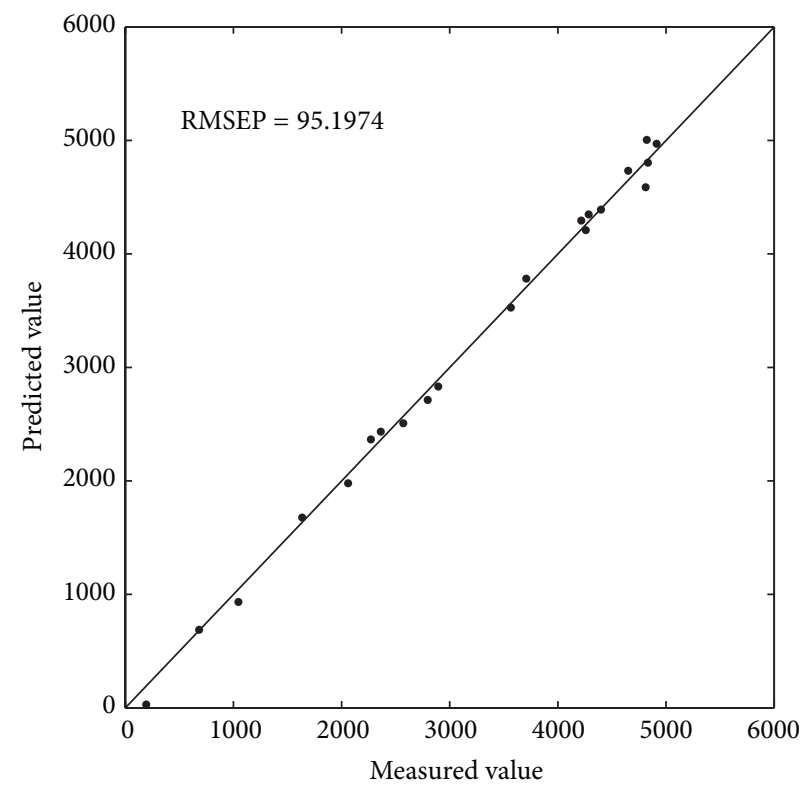

(b)

FIGURE 2: Prediction value versus measured value scatter diagram of different methods for $\mathrm{CH}_{4}$. (a) PLS; (b) RBFEI-PLS.

value versus predicted value of PLS and RBFEI-PLS for $\mathrm{CO}_{2}$. Figure 4(a) shows that most points are distributed on both sides of the diagonal line and some points are even distant from the diagonal line. In Figure 4(b), most points of RBFEIPLS lie on the diagonal line. As a result, the RBFEI-PLS method has a higher predictive accuracy.

In summary, the experiments results verify that RBFEIPLS could be adopted for quantitative analysis of flue gas of natural gas combustion successfully and has higher predictive capability. 


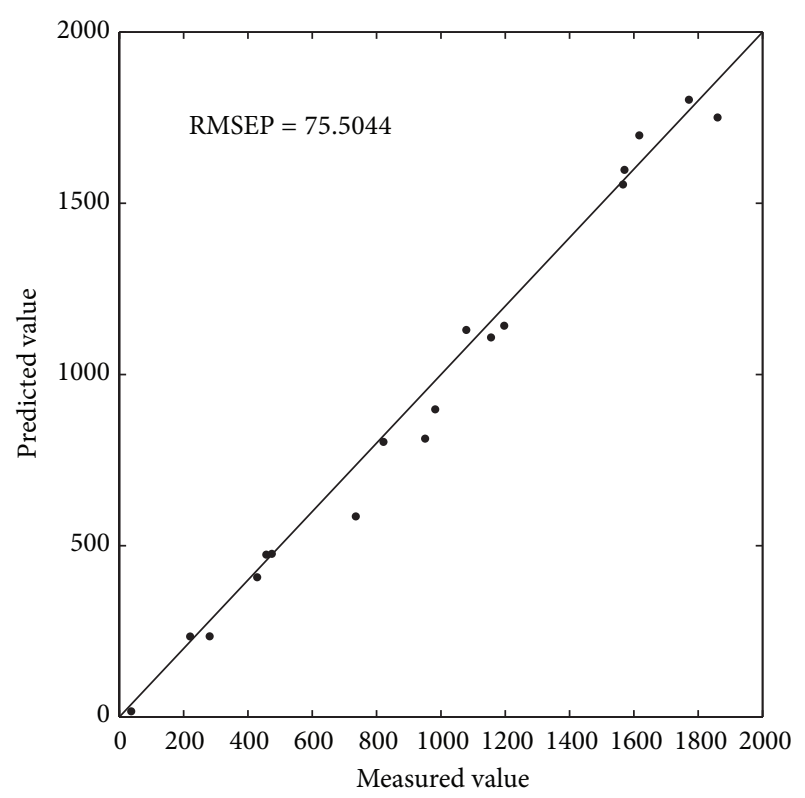

(a)

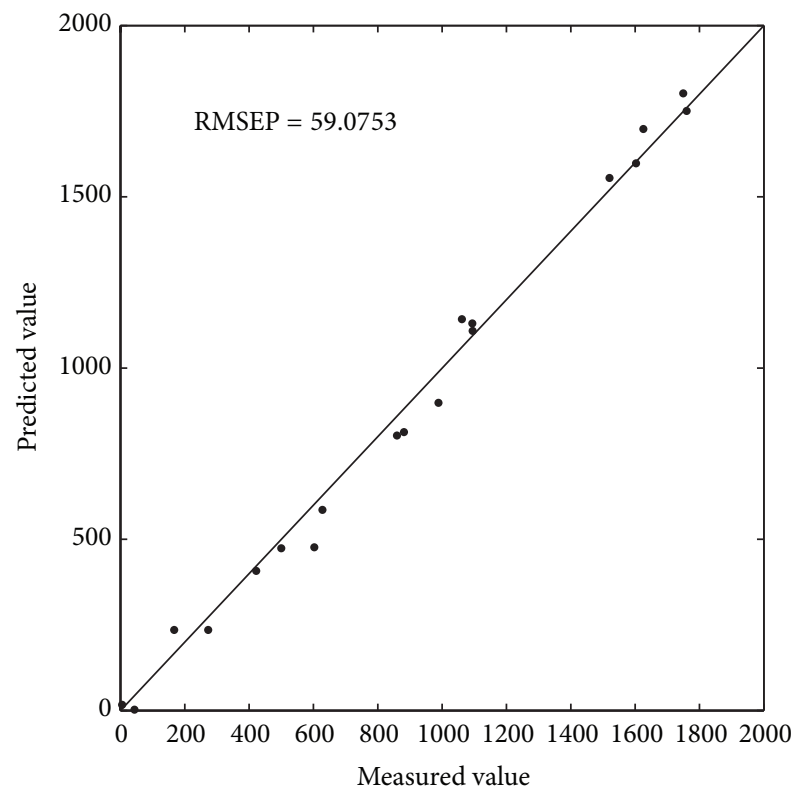

(b)

Figure 3: Prediction value versus measured value scatter diagram of different methods for CO. (a) PLS; (b) RBFEI-PLS.

\section{Conclusions}

In this paper, a component prediction method which combines RBFNN and NPLS with extended input is proposed for component prediction of flue gas of natural gas combustion. Since the proposed method uses RBFNN to extend the input matrix, the nonlinear-related spectral data could be dealt with for quantitative analysis. The experiments results verify that the prediction capability of the proposed method is higher for various components of the flue gas and that the RMSEP values of the proposed method for methane, carbon monoxide, and

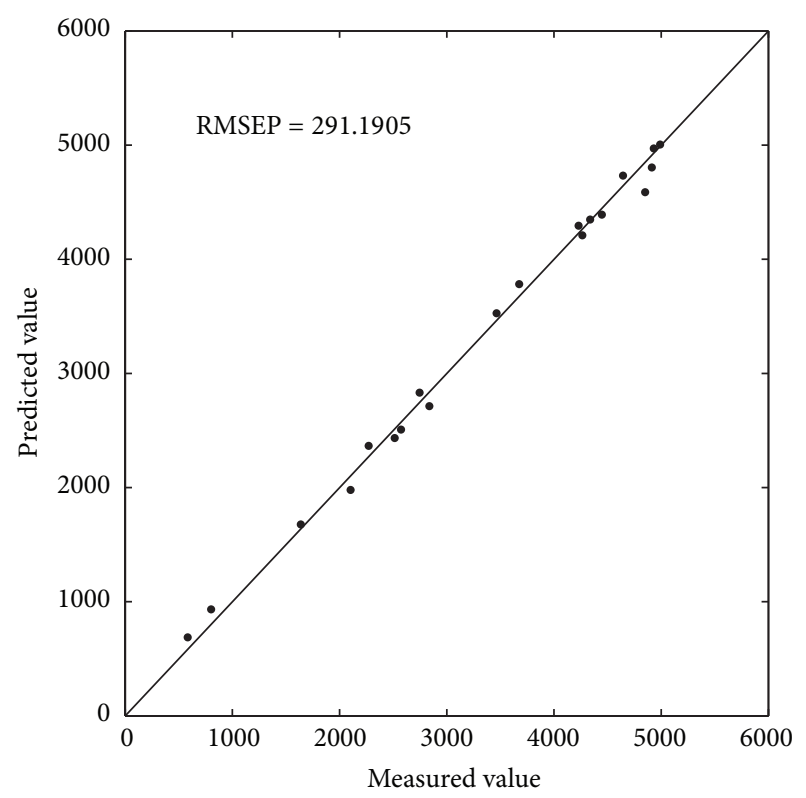

(a)

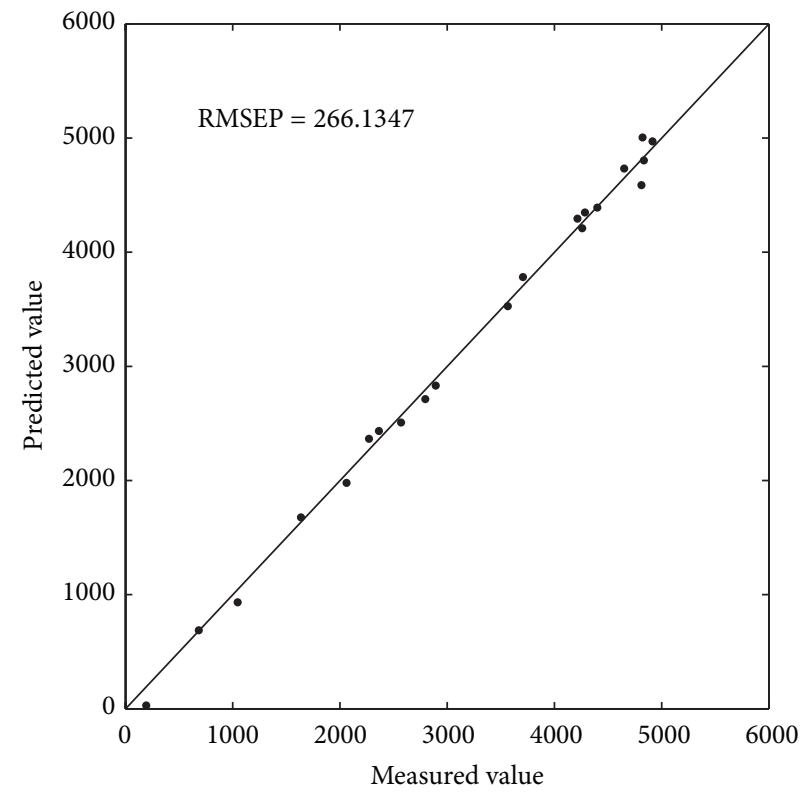

(b)

FIGURE 4: Prediction value versus measured value scatter diagram of different methods for $\mathrm{CO}_{2}$. (a) PLS; (b) RBFEI-PLS.

carbon dioxide are, respectively, reduced by $4.74 \%, 21.76 \%$, and $5.32 \%$ compared to those of PLS. Therefore, the proposed method is an accurate and practical component prediction method for flue gas of natural gas combustion and could be applied for spectral analyses of other analysts.

\section{Conflict of Interests}

The authors declare that there is no conflict of interests regarding the publication of this paper. 


\section{Acknowledgments}

This work is supported by the National Natural Science Foundation of China (61375055), the Program for New Century Excellent Talents in University (NCET-12-0447), and the Fundamental Research Funds for the Central University.

\section{References}

[1] R. Carapellucci and L. Giordano, "A comparison between exergetic and economic criteria for optimizing the heat recovery steam generators of gas-steam power plants," Energy, vol. 58, pp. 458-472, 2013.

[2] G. Hassan, M. Pourkashanian, D. Ingham, L. Ma, P. Newman, and A. Odedra, "Predictions of $\mathrm{CO}$ and $\mathrm{NO}_{x}$ emissions from steam cracking furnaces using GRI2. 11 detailed reaction mechanism-a CFD investigation," Computers \& Chemical Engineering, vol. 58, pp. 68-83, 2013.

[3] G. Li, Z. Li, M. J. Wang, L. Lin, and B. J. Zhang, "Noninvasive measurement of serum bilirubin employing near-infrared spectroscopy," Chinese Journal of Analytical Chemistry, vol. 41, pp. 263-267, 2013.

[4] C. Schaefer, C. Lecomte, D. Clicq, A. Merschaert, E. Norrant, and F. Fotiadu, "On-line near infrared spectroscopy as a Process Analytical Technology (PAT) tool to control an industrial seeded API crystallization," Journal of Pharmaceutical and Biomedical Analysis, vol. 83, pp. 194-201, 2013.

[5] R. Yang, R. Liu, K. Xu, and Y. Yang, "Discrimination of adulterated milk using NPLS-DA combined with two-dimensional correlation near-infrared spectroscopy," Acta Photonica Sinica, vol. 42, pp. 580-585, 2013.

[6] H. Namkung, J. Kim, H. Chung, and M. A. Arnold, "Impact of pellet thickness on quantitative terahertz spectroscopy of solid samples in a polyethylene matrix," Analytical Chemistry, vol. 85, pp. 3674-3681, 2013.

[7] P. Geladi and B. R. Kowalski, "Partial least-squares regression: a tutorial," Analytica Chimica Acta, vol. 185, pp. 1-17, 1986.

[8] K. X. Qiu, X. Z. Song, G. Tang, L. J. Wu, and S. G. Min, "Determination of fipronil in acetamiprid formulation by attenuated total reflectance-mid-infrared spectroscopy combined with partial least squares regression," Analytical Letters, vol. 46, pp. 2388-2399, 2013.

[9] X. Gang and S. Wen-qi, "Research on feasibility of rapid determination of the moisture content in ABC dry chemical using near infrared spectroscopy," Acta Photonica Sinica, vol. 42, pp. 908-911, 2013.

[10] B. Álvarez Sánchez, F. Priego Capote, J. García Olmo, M. C. Ortiz Fernández, L. A. Sarabia Peinador, and M. D. Luque De Castro, "Near-infrared spectroscopy and partial least squaresclass modeling (PLS-CM) for metabolomics fingerprinting discrimination of intervention breakfasts ingested by obese individuals," Journal of Chemometrics, vol. 27, pp. 221-232, 2013.

[11] S. Wold, N. Kettaneh-Wold, and B. Skagerberg, "Nonlinear PLS modeling," Chemometrics and Intelligent Laboratory Systems, vol. 7, no. 1-2, pp. 53-65, 1989.

[12] E. C. Malthouse, A. C. Tamhane, and R. S. H. Mah, "Nonlinear partial least squares," Computers and Chemical Engineering, vol. 21, no. 8, pp. 875-890, 1997.

[13] J. Liu, K. Min, C. Han, and K. S. Chang, "Robust nonlinear PLS based on neural networks and application to composition estimator for high-purity distillation columns," Korean Journal of Chemical Engineering, vol. 17, no. 2, pp. 184-192, 2000.
[14] K. P. Singh, P. Ojha, A. Malik, and G. Jain, "Partial least squares and artificial neural networks modeling for predicting chlorophenol removal from aqueous solution," Chemometrics and Intelligent Laboratory Systems, vol. 99, no. 2, pp. 150-160, 2009.

[15] R.-D. Jia, Z.-Z. Mao, Y.-Q. Chang, and J.-W. Zhou, "Nonlinear robust partial least squares based on projection pursuit and its application," Control Theory and Applications, vol. 27, no. 3, pp. 391-399, 2010.

[16] S. Lee and W. S. Choi, "A multi-industry bankruptcy prediction model using back-propagation neural network and multivariate discriminant analysis," Expert Systems with Applications, vol. 40, pp. 2941-2946, 2013.

[17] R. Cui, S. S. Ge, B. Voon Ee How, and Y. Sang Choo, "Leaderfollower formation control of underactuated autonomous underwater vehicles," Ocean Engineering, vol. 37, no. 17-18, pp. 1491-1502, 2010.

[18] Z. Li and C. Yang, "Neural-adaptive output feedback control of a class of transportation vehicles based on wheeled inverted pendulum models," IEEE Transactions on Control Systems Technology, vol. 20, pp. 1583-1591, 2012.

[19] G. E. Tsekouras and J. Tsimikas, "On training RBF neural networks using input-output fuzzy clustering and particle swarm optimization," Fuzzy Sets and Systems, vol. 221, pp. 65-89, 2013.

[20] T. Naes, T. Isaksson, and B. Kowalski, "Locally weighted regression and scatter correction for near-infrared reflectance data," Analytical Chemistry, vol. 62, no. 7, pp. 664-673, 1990. 

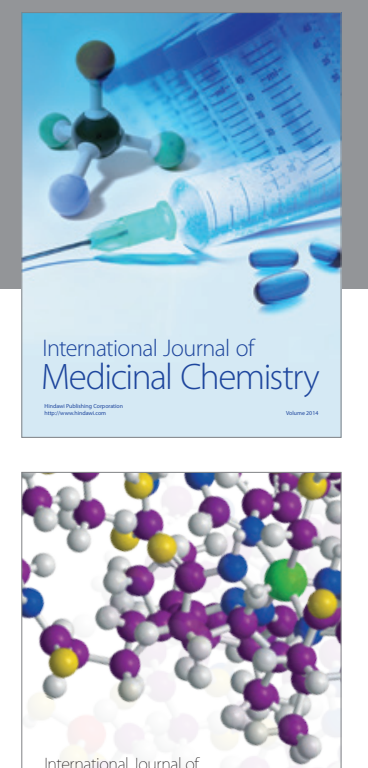

\section{Carbohydrate} Chemistry

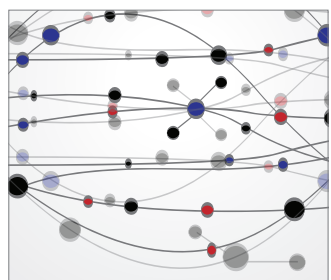

The Scientific World Journal
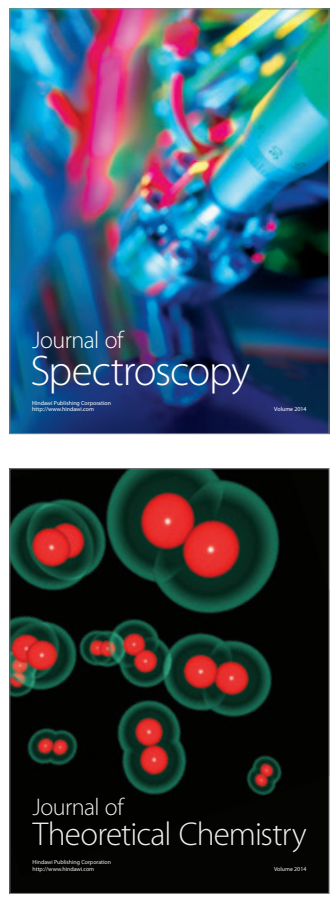
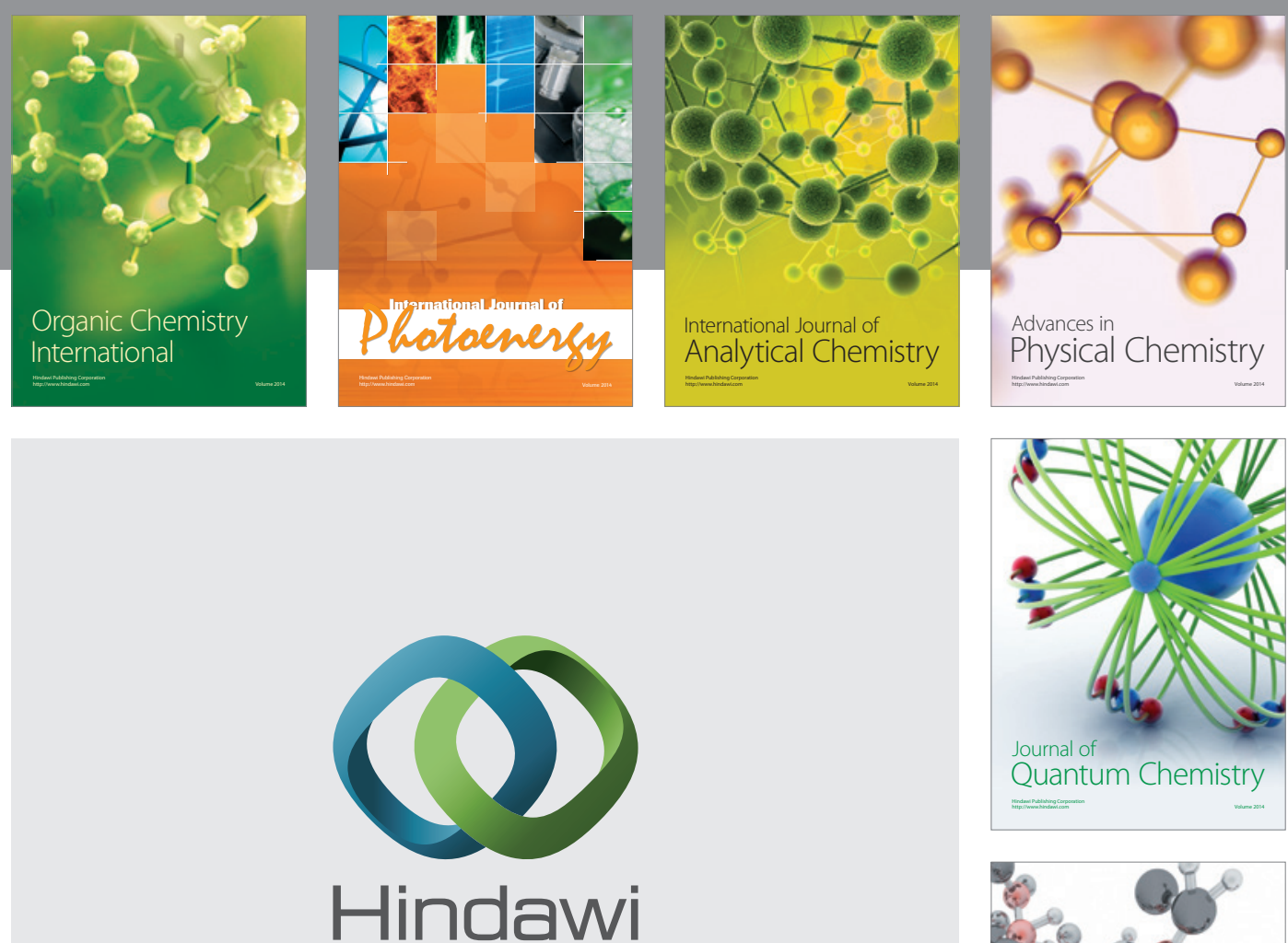

Submit your manuscripts at

http://www.hindawi.com

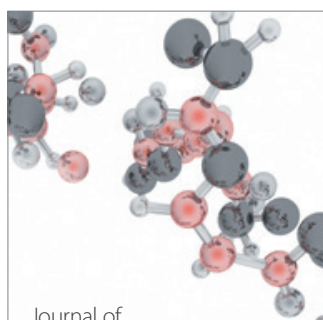

Analytical Methods

in Chemistry

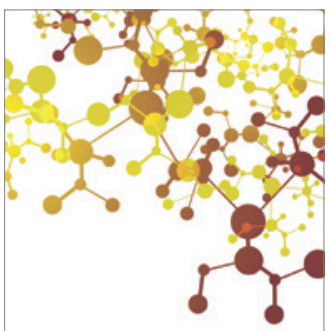

Journal of

Applied Chemistry

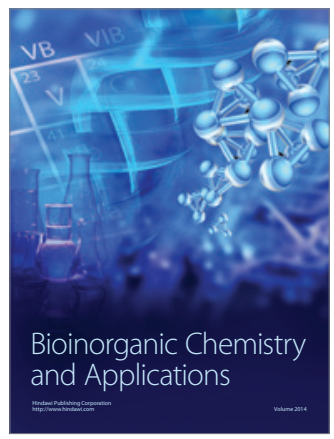

Inorganic Chemistry
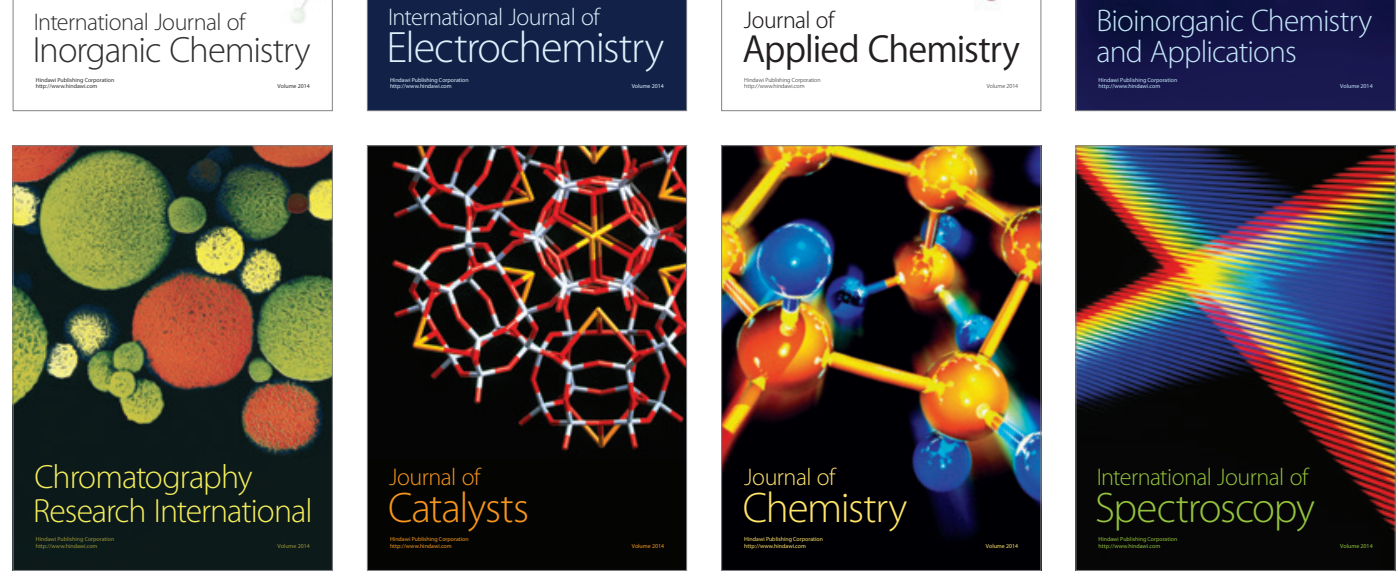